\title{
MRI characteristics in acute ischemic stroke patients with preceding direct oral anticoagulant therapy as compared to vitamin $\mathrm{K}$ antagonists
}

Thomas Raphael Meinel ${ }^{1 *}$ (D), Johannes Kaesmacher², Jan Gralla³, David J. Seiffge ${ }^{1}$, Elias Auer ${ }^{1}$, Sebastién Frey ${ }^{1}$, Marwan El-Koussy ${ }^{3}$, Marcel Arnold ${ }^{1}$, Urs Fischer ${ }^{1}$, Martina Göldlin ${ }^{1,3}$, Simon Jung ${ }^{1+}$ and Arsany Hakim ${ }^{3+}$

\begin{abstract}
Background: Despite the utility of neuroimaging in the diagnostic and therapeutic management of patients with acute ischemic stroke (AIS), imaging characteristics in patients with preceding direct oral anticoagulants (DOAC) compared to vitamin $\mathrm{K}$ antagonists (VKA) have hardly been described. We aimed to determine presence of large vessel occlusion (LVO), thrombus length, infarction diameter, and occurrence of hemorrhagic transformation in AIS patients with preceding DOAC as compared to VKA therapy.

Methods: Using a prospectively collected cohort of AIS patients, we performed univariate and multivariable regression analyses regarding imaging outcomes. Additionally, we provide a sensitivity analysis for the subgroup of patients with confirmed therapeutic anticoagulation.

Results: We included AIS in patients with preceding DOAC $(N=75)$ and VKA $(N=61)$ therapy, median age 79 (IQR $70-83), 39 \%$ female. Presence of any LVO between DOAC and VKA patients (29.3\% versus $37.7 \%, P=0.361)$, and target LVO for endovascular therapy $(26.7 \%$ versus $27.9 \%, P=1.0)$ was equal with a similar occlusion pattern. DOAC as compared to VKA were associated with a similar rate of target LVO for EVT (aOR 0.835, 95\% Cl 0.368-1.898). The presence of multiple lesions and characteristics of the thrombus were similar in DOAC and VKA patients. Acute ischemic lesion diameter in real world patients was equal in patients taking DOAC as compared to VKA. Lesion diameter in VKA patients (median $13 \mathrm{~mm}$, IQR 6-26 versus median $20 \mathrm{~mm}$, IQR 7-36, $P=0.001$ ), but not DOAC patients was smaller in the setting of confirmed therapeutic VKA. The frequency of radiological hemorrhagic transformation and symptomatic intracranial hemorrhage in OAC patients was low. Sensitivity analysis considering only patients with confirmed therapeutic anticoagulation did not change any of the results.
\end{abstract}

Conclusion: Preceding DOAC treatment showed equal rates of LVO and infarct size as compared to VKA in AIS patients. This study adds to the knowledge of imaging findings in AIS patients with preceding anticoagulation.

Keywords: Acute ischemic stroke, DOAC, VKA, Large vessel occlusion, Anticoagulation, Infarction size, Hemorrhagic transformation

\footnotetext{
* Correspondence: thomas.meinel@insel.ch

${ }^{\dagger}$ Simon Jung and Arsany Hakim contributed equally to this work.

${ }^{1}$ Department of Neurology, Inselspital, Bern University Hospital, University of Bern, Freiburgstrasse 8, CH-3010 Bern, Switzerland

Full list of author information is available at the end of the article
}

C C The Author(s). 2020 Open Access This article is licensed under a Creative Commons Attribution 4.0 International License, which permits use, sharing, adaptation, distribution and reproduction in any medium or format, as long as you give appropriate credit to the original author(s) and the source, provide a link to the Creative Commons licence, and indicate if changes were made. The images or other third party material in this article are included in the article's Creative Commons. licence, unless indicated otherwise in a credit line to the material. If material is not included in the article's Creative Commons licence and your intended use is not permitted by statutory regulation or exceeds the permitted use, you will need to obtain permission directly from the copyright holder. To view a copy of this licence, visit http://creativecommons.org/licenses/by/4.0/ The Creative Commons Public Domain Dedication waiver (http://creativecommons.org/publicdomain/zero/1.0/) applies to the data made available in this article, unless otherwise stated in a credit line to the data. 


\section{Background}

Patients with atrial fibrillation (AF) have a five-fold increased risk of acute ischemic stroke (AIS). Therefore, oral anticoagulation $(\mathrm{OAC})$ is recommended after risk stratification to prevent thromboembolic events [1]. Due to the increased risk of intracerebral hemorrhage and downsides of management of Vitamin $\mathrm{K}$ antagonists (VKA), direct oral anticoagulants (DOAC) are now recommended as first line $\mathrm{OAC}$ in patients with $\mathrm{AF}[1,2]$. Even with the pronounced preventive reduction of AIS by OAC [1], AIS occurs with an estimated rate of about $1-2 \%$ per year in patients taking a DOAC [3].

Initial imaging in AIS is crucial to rapidly obtain an accurate diagnosis, select patients for acute recanalization treatments, predict outcome, guide etiological work-up and initiate early secondary prevention [4-6]. Nevertheless, data on imaging characteristics of AIS in patients taking OAC, especially DOAC are sparse $[7,8]$. Few comparative small studies looked at size, volume and presence of a large vessel occlusion (LVO) $[9,10]$ in patients taking DOAC versus VKA, with preliminary results in favor of DOAC. However, no laboratory assessment of OAC activity or information on compliance for DOAC was available.

Hence, we aimed to explore the frequency of any LVO or target LVO for endovascular stroke treatment and other imaging characteristics of AIS in a prospectively collected registry of patients admitted to our comprehensive university stroke center prescribed with OAC. Additionally, we present a sensitivity analysis considering only patients with confirmed therapeutic DOAC and VKA activity on admission.

\section{Methods}

Inclusion criteria for this study were a diagnosis of AIS and preceding $\mathrm{OAC}$ prescription at the time point of symptom onset (either DOAC or VKA). We selected a random sample of patients fulfilling the inclusion criteria from our prospectively consecutive local stroke registry from February 2015 to August 2017. We chose random sampling because of lack of capacity to analyze the images of all DOAC and VKA patients of the registry. For this purpose, a random number between 0 and 1 was generated using the excel function = RAND for each patient. By sorting the random number ascendingly, the first 80 patients were included in the study. We analyzed only patients with MRI on admission, which represents the preferred imaging modality in our center (about 60$70 \%)$. We excluded patients with transient ischemic attacks, stroke mimics, non-cerebral ischemic events, refusal of further use of biological data and missing information on OAC medication from this analysis. Antiplatelet prescription did not affect group assignment. For the sensitivity analysis, we further defined confirmed therapeutic OAC as specific drug activity >
$50 \mathrm{ng} / \mathrm{ml}$ in patients taking DOAC [11-15] and INR > 1.7 in VKA patients. If no specific drug activity was available in patients taking DOAC, confirmed therapeutic OAC was defined as reported previously [16].

We assessed the following information from the registry and in case of missing items in the medical records: demographic variables (age, sex, prestroke modified Rankin Scale (mRS)), cardiovascular risk factors, clinical parameters (blood pressure, National Institutes of Health Stroke Scale (NIHSS), onset type, TOAST-etiology, antithrombotic, antihypertensive and lipid lowering medication before admission, laboratory parameters (cholesterol, glucose, creatinine, and international normalized ratio INR) and acute recanalization treatment such as intravenous thrombolysis (IVT) and endovascular therapy (EVT).

\section{Imaging protocol}

We acquired MRI scans on the day of admission, either on a $1.5 \mathrm{~T}$ scanner (Siemens Magnetom Avanto and Siemens Magnetom Aera, Siemens Medical Solutions, Erlangen, Germany) or a $3 \mathrm{~T}$ system (Siemens Magnetom Verio, Siemens Medical Solutions, Erlangen, Germany). Our MRI protocol includes axial diffusion-weighted imaging (DWI) with apparent diffusion coefficient (ADC) (5 $\mathrm{mm}$ slice thickness), Fluid-attenuated inversion recovery (FLAIR) (5 mm slice thickness), susceptibility-weighted imaging (SWI) (1.6 mm slice thickness), and a time of flight (TOF) angiography (0.5 mm slice thickness). After application of intravenous Gadobutrol (Gadovist; Bayer Schering Pharma, Berlin, Germany) in an antecubital vein with a $5 \mathrm{ml} / \mathrm{s}$ injection rate, we acquired a standard dynamic susceptibility contrast (DSC) MRI perfusion (Tmax, $5 \mathrm{~mm}$ slice thickness) as well as a contrast enhanced T1weighted sequence (slice thickness $5 \mathrm{~mm}$ ). Finally, a contrast enhanced magnetic resonance angiography (CE MRA) of the head and neck vessels was acquired after injection of a second bolus of Gadobutrol with a $3 \mathrm{ml} / \mathrm{s}$ injection rate. Post-processing of DSC was performed using Olea sphere v.2.3 (Olea Medical, La Ciotat, France), using deconvolution method with oscillant singular value decomposition. Follow-up imaging was performed using the same scanners and same MRI protocol.

\section{Outcomes and imaging analysis}

Two raters (EA and SF) assessed the imaging outcomes after a standardized training and blinded to patients' OAC status. All ratings were controlled by a board-certified neuroradiologist $(\mathrm{AH})$ with more than 10 years' experience in stroke imaging. The primary outcome was the presence of any LVO defined as occlusion of an artery supplying the brain visible on TOF or contrast enhanced MRangiography. Secondary outcomes included: target LVO for EVT defined as LVO until or proximal to A1/M2-segment of the anterior circulation or P1-segment of the posterior 
circulation; presence and length of thrombus as determined by SWI; size of hypoperfusion; degree of DWI infarction core and hypoperfused area mismatch defined as small mismatch (infarction core $\geq 2 / 3$ of the hypoperfused region), moderate mismatch (infarction core $>1 / 3$ and $<2 / 3$ of the hypoperfused region) and large mismatch (infarction core $\leq 1 / 3$ of the hypoperfused region) by visual judgment; maximal diameter in $\mathrm{mm}$ of the acute ischemic lesion as determined in DWI (axis 1), maximal diameter of a perpendicular axis of the acute ischemic lesion (axis 2). In case of multiple lesions, the largest lesion was analyzed for the primary analysis. Other outcomes included size of ischemia demarcation in FLAIR sequence at $24 \mathrm{~h}$; presence of cerebral microbleeds (CMB) according to established criteria at baseline [17]; presence of hemorrhagic transformation at $24 \mathrm{~h}$ according to the Heidelberg classification [18]; presence of more than one (multiple) acute ischemic lesions and severity of white matter hyperintensities according to the Fazekas score [19].

\section{Statistics}

We compared the two OAC groups (DOAC versus VKA), and patients with confirmed and infra-therapeutic OAC using appropriate statistical measures (Fisher's exact test for categorical variables, Mann-Whitney-U-Test for nonnormally continuous or ordinally scaled variables, and Welch's t-test for independent normally distributed data). We determined published predictors of stroke severity as pre-specified covariates and factors for the multivariate analysis avoiding collinearity. For the primary analysis, the association of OAC type (DOAC versus VKA) with any LVO or target LVO for EVT was assessed using binary logistic regression adjusting for the following confounders: age (continuous), sex (categorical), admission glucose (linear, adjusted odds ratio (aOR) per $\mathrm{mmol} / \mathrm{L}$ increase), arterial hypertension (categorical) and atrial fibrillation (categorical). For the secondary analysis, the same confounders were included in a multivariable linear regression analysis. Patients with missing data items were excluded from the multivariate analysis. For the sensitivity analysis, the same model was used considering only patients with confirmed therapeutic OAC activity on admission. We used a level of significance of 0.05 .

\section{Results}

The random sample of OAC patients with AIS fulfilling the in- and exclusion criteria included 75 DOAC patients (59 Rivaroxaban, 12 Apixaban, 4 Dabigatran) and 61 VKA patients. Either documented information on compliance or a reliable assessment of DOAC activity was available in $45 / 75(60 \%)$ of patients taking DOAC. INR was available in all patients taking VKA. OAC activity was confirmed therapeutic in 35/75 (46.7\%) of DOAC patients and 45/61 (73.8\%) of VKA patients. Table 1 summarizes the demographic and clinical data of the patients. Patients with preceding DOAC prescription were younger and had less severe white matter lesions as compared to VKA patients. Otherwise, groups were comparable for baseline and treatment variables.

There was no difference in the frequency of any LVO between DOAC and VKA patients (29.3\% versus 37.7\%, $P=0.361$ ) on univariate analysis. There was also no difference in target LVO for EVT between DOAC and VKA patients $(26.7 \%$ versus $27.9 \%, P=1.0)$, but the size of the initial hypoperfusion was significantly larger in DOAC patients $(80 \mathrm{~mm}$, IQR $38-118$ versus $49 \mathrm{~mm}$, IQR 36-67; $P=0.039$ ). The occlusion pattern was similar in patients with DOAC and VKA (Table 2) without a signal for more proximal or distal occlusions in either group. Equally, on binary logistic regression analysis DOAC as compared to VKA was not associated with the presence of any LVO (aOR 0.622 , 95\% CI $0.283-1.366$ ) or presence of a target LVO for EVT (aOR 0.835, 95\% CI 0.368-1.898).

Sensitivity analysis considering only patients with confirmed therapeutic OAC did not change those results. The frequency of DWI/Perfusion mismatch was equal between groups. In those patients with any vessel occlusion, SWI could visualize the thrombus in 19/22 (86\%) of DOAC patients (median $8 \mathrm{~mm}$, IQR $6-20$ ) and $57 \%$ of VKA patients (median $13 \mathrm{~mm}$, IQR 7-24).

For the secondary outcomes, maximal acute DWI lesion diameter in DOAC patients (median 18, IQR 11-36) was equal to VKA (median 20, IQR 7-36) on univariate analysis $(P=0.607$, Table 3$)$. Also, according to the multivariable linear regression analysis adjusting for confounders as outlined in the methods section, VKA was not significantly associated with increased acute DWI lesion diameter $(\beta$ 2.385, 95\%-CI $-10.070-14.840, P=0.705$ ) as compared to DOAC. Both analyses remained unchanged when only considering patients with confirmed therapeutic OAC activity. There was no difference in the maximal diameter of the perpendicular axis of the acute ischemic lesion. In VKA patients, acute DWI lesion diameter was smaller in the case of confirmed therapeutic OAC activity. For DOAC patients, this effect could not be seen (Fig. 1).

Size of ischemia lesion diameter at $24 \mathrm{~h}$ (FLAIR sequence) was equal in DOAC patients (median $18 \mathrm{~mm}$, IQR 12-38) and VKA patients (median $27 \mathrm{~mm}$, IQR 8$50, P=0.409)$. The rate of multiple lesions was equal between groups.

At baseline, $38.9 \%$ of DOAC patients and $47.4 \%$ of VKA patients had at least one CMB. One patient (1.3\%) in the DOAC group (Heidelberg class 3 ) and one patient $(1.6 \%)$ in the VKA group (Heidelberg class 2) suffered symptomatic intracranial hemorrhage at $24 \mathrm{~h}$. 5/75 (6.7\%) of DOAC patients and 10/61 (11.5\%) of VKA patients showed asymptomatic hemorrhagic transformation of the ischemic lesion at $24 \mathrm{~h}$. 
Table 1 Baseline characteristics comparing patients with DOAC and VKA pretreatment

\begin{tabular}{|c|c|c|c|c|c|c|}
\hline & $\begin{array}{l}\text { DOAC pretreatment } \\
(\boldsymbol{N}=75)\end{array}$ & $\begin{array}{l}\text { VKA pretreatment } \\
(\boldsymbol{N}=61)\end{array}$ & $P$ & $\begin{array}{l}\text { Confirmed therapeutic } \\
\text { DOAC }(\boldsymbol{N}=35)\end{array}$ & $\begin{array}{l}\text { Confirmed therapeutic } \\
\text { VKA }(\boldsymbol{N}=45)\end{array}$ & $P$ \\
\hline \multicolumn{7}{|l|}{ Clinical items } \\
\hline Age (years) & $76(67-82)$ & $81(75-84)$ & 0.010 & $76(68-83)$ & $80(74-84)$ & 0.117 \\
\hline Body mass index (kg/m2) & $26.4(22.9-29.4)$ & $25.8(23.6-28.6)$ & 0.674 & $27.0(23.1-30.0)$ & $25.7(23.7-29.3)$ & 0.376 \\
\hline Sex (female) & 27/75 (36.0\%) & $26 / 61(42.6 \%)$ & 0.482 & 13/35 (37.1\%) & 20/45 (44.4\%) & 0.648 \\
\hline Preevent modified Rankin Scale & $0.5(0-1)$ & $1(0-2)$ & 0.306 & $0(0-1)$ & $1(0-2)$ & 0.327 \\
\hline Onset & & & 0.398 & & & 0.407 \\
\hline - Known & $34 / 75(45.3 \%)$ & $36 / 61(59.0 \%)$ & & 18/35 (51.4\%) & 29/45 (64.4\%) & \\
\hline - Wake-up & 14/75 (18.7\%) & $7 / 61(11.5 \%)$ & & $6 / 35(17.1 \%)$ & 4/45 (8.9\%) & \\
\hline - Unknown & $27 / 75(36.0 \%)$ & 18/61 (29.5\%) & & $11 / 35(31.4 \%)$ & $12 / 45(26.7 \%)$ & \\
\hline 1st blood pressure, systolic & $160(136-176)$ & $160(146-182)$ & 0.318 & $156(135-178)$ & $159(143-176)$ & 0.446 \\
\hline 1st blood pressure, diastolic & 79 (70-94) & $88(73-100)$ & 0.089 & $78(69-92)$ & 87 (72-99) & 0.145 \\
\hline 1st Glucose (mmol/L) & $6.2(5.5-7.4)$ & $6.5(5.5-7.9)$ & 0.500 & $6.3(5.7-7.7)$ & $6.6(5.6-7.9)$ & 0.773 \\
\hline 1st Cholesterol, total (mmol/L) & $4.4(3.8-5.1)$ & $4.5(4.0-5.3)$ & 0.244 & $4.5(3.8-5.3)$ & $4.5(3.8-5.2)$ & 0.848 \\
\hline 1st Cholesterol, LDL (mmol/L) & $2.4(1.9-3.2)$ & $2.4(2.0-3.3)$ & 0.570 & $2.4(1.9-3.3)$ & $2.4(2.1-3.4)$ & 0.587 \\
\hline 1st Creatinine (umol/L) & $87(75-102)$ & $88(72-105)$ & 0.666 & $89(75-101)$ & $86(70-107)$ & 0.809 \\
\hline NIHSS on admission & $3(2-8)$ & $5(1-10)$ & 0.697 & $4.5(2-8.5)$ & $5(1-12)$ & 0.996 \\
\hline \multicolumn{7}{|l|}{ Medication } \\
\hline Additional Antiplatelet & 11/75 (14.7\%) & 11/61 (18.3\%) & 0.642 & $3 / 35(8.6 \%)$ & 9/45 (20\%) & 0.210 \\
\hline Lipid lowering drug & $31 / 75(41.3 \%)$ & 26/61 (42.6\%) & 0.661 & $16 / 35(45.7 \%)$ & $22 / 45(48.9 \%)$ & 0.824 \\
\hline \multicolumn{7}{|l|}{ Anticoagulation } \\
\hline - Confirmed therapeutic & $35 / 75(46.7 \%)$ & 45/61 (73.8\%) & & & & \\
\hline - Uncertain & $30 / 75(40 \%)$ & 0 & & & & \\
\hline - Confirmed non-therapeutic & 10/75 (13.3\%) & 16/61 (26.2\%) & & & & \\
\hline \multicolumn{7}{|l|}{ Risk factors } \\
\hline Previous stroke & 22/75 (29.3\%) & $14 / 61(23.0 \%)$ & 0.626 & $11 / 35(31.4 \%)$ & $9 / 45(20 \%)$ & 0.239 \\
\hline Previous transient ischemic attack & $8 / 75(10.7 \%)$ & $7 / 61(11.5 \%)$ & 0.913 & 6/35 (17.1\%) & $6 / 45(13.3 \%)$ & 0.454 \\
\hline Arterial Hypertension & $62 / 75(82.7 \%)$ & $53 / 61(86.9 \%)$ & 0.690 & 28/35 (90\%) & 40/45 (88.9\%) & 0.370 \\
\hline Diabetes & 15/75 (20.0\%) & 13/61 (21.3\%) & 0.957 & $7 / 35(20 \%)$ & 9/45 (20\%) & 0.984 \\
\hline Hyperlipidemia & $46 / 75(61.3 \%)$ & $41 / 61(67.2 \%)$ & 0.623 & 19/35 (54.3\%) & $30 / 45(66.7 \%)$ & 0.192 \\
\hline Smoking & 15/75 (20.0\%) & $4 / 61(6.6 \%)$ & 0.073 & $7 / 35(20 \%)$ & 2/45 (4.4\%) & 0.076 \\
\hline Heart failure & $11 / 75(14.7 \%)$ & 15/61 (24.6\%) & 0.328 & $5 / 35(14.3 \%)$ & $11 / 45(24.4 \%)$ & 0.296 \\
\hline Atrial Fibrillation & $52 / 75(69.3 \%)$ & $41 / 61(67.2 \%)$ & 0.617 & 23/35 (65.7\%) & $31 / 45(68.9 \%)$ & 0.764 \\
\hline Mechanical Heart Valve & 0 & $4 / 61(6.6 \%)$ & 0.098 & 0 & 3/45 (6.7\%) & 0.135 \\
\hline Low Ejection Fraction (<30\%) & $2 / 75(2.7 \%)$ & $1 / 61(1.6 \%)$ & 0.657 & $1 / 35(2.9 \%)$ & 0 & 0.437 \\
\hline Peripheral artery disease & $8 / 75(10.7 \%)$ & $4 / 61(6.6 \%)$ & 0.677 & $3 / 35(8.6 \%)$ & 4/45 (8.9\%) & 0.983 \\
\hline \multicolumn{7}{|l|}{ Treatment } \\
\hline Acute recanalization therapy & & & 0.905 & & & 0.464 \\
\hline - Intravenous thrombolysis & $2 / 75(2.7 \%)$ & $1 / 61(1.6 \%)$ & & $1 / 35(2.9 \%)$ & 0 & \\
\hline - Endovascular thrombectomy & 10/75 (13.3\%) & 10/61 (16.4\%) & & 3/35 (8.6\%) & 6/45 (13.3\%) & \\
\hline - Intraarterial thrombolysis & $1 / 75(1.3 \%)$ & $1 / 61(1.6 \%)$ & & 0 & $1 / 45(2.2 \%)$ & \\
\hline \multicolumn{7}{|l|}{ Imaging } \\
\hline Fazekas Score & $1(0-2)$ & $2(1-3)$ & $<0.001$ & $1(0-2)$ & $2(1-3)$ & 0.014 \\
\hline Cerebral Microbleeds present & 28/72 (38.9\%) & 27/57 (47.4\%) & 0.373 & 16/33 (48.5\%) & $22 / 42(52.4 \%)$ & 0.818 \\
\hline
\end{tabular}


Table 2 Patterns of Large Vessel Occlusions comparing patients with DOAC and VKA pretreatment

\begin{tabular}{|c|c|c|c|c|c|c|}
\hline & $\begin{array}{l}\text { DOAC pretreatment } \\
(\boldsymbol{N}=75)\end{array}$ & $\begin{array}{l}\text { VKA pretreatment } \\
(\boldsymbol{N}=61)\end{array}$ & $P$ & $\begin{array}{l}\text { Confirmed therapeutic } \\
\text { DOAC }(\boldsymbol{N}=35)\end{array}$ & $\begin{array}{l}\text { Confirmed therapeutic } \\
\text { VKA }(\boldsymbol{N}=45)\end{array}$ & $P$ \\
\hline Any large vessel occlusion & 22/75 (29.3\%) & 23/61 (37.7\%) & 0.361 & 9/35 (25.7\%) & $14 / 45(31.1 \%)$ & 0.628 \\
\hline - Proximal ICA & $1(4.5 \%)$ & $1(4.3 \%)$ & & 0 & 0 & \\
\hline - Carotid-T & $2(9.1 \%)$ & $2(8.7 \%)$ & & $1(11.1 \%)$ & $1(7.1 \%)$ & \\
\hline - Proximal M1 & $7(31.8 \%)$ & $4(17.4 \%)$ & & $3(33.3 \%)$ & $1(7.1 \%)$ & \\
\hline - Distal M1 & $3(13.6 \%)$ & $1(4.3 \%)$ & & $1(11.1 \%)$ & $1(7.1 \%)$ & \\
\hline$-\mathrm{M} 2$ & $6(27.3 \%)$ & $6(26.1 \%)$ & & $3(33.3 \%)$ & $4(28.6 \%)$ & \\
\hline$-\mathrm{M3}$ & 0 & $1(4.3 \%)$ & & 0 & $1(7.1 \%)$ & \\
\hline$-A 1$ & 0 & 0 & & 0 & 0 & \\
\hline$-A 2$ & $1(4.5 \%)$ & 0 & & 0 & 0 & \\
\hline$-V_{4}$ & 0 & $1(4.3 \%)$ & & 0 & $1(7.1 \%)$ & \\
\hline$-B A$ & 0 & 0 & & 0 & 0 & \\
\hline$-\mathrm{P} 1$ & 0 & $1(4.3 \%)$ & & 0 & $1(7.1 \%)$ & \\
\hline$-P 2 / 3$ & $1(4.5 \%)$ & $4(17.4 \%)$ & & 0 & $4(28.6 \%)$ & \\
\hline - Multiple & $1(4.5 \%)$ & $1(4.3 \%)$ & & $1(11.1 \%)$ & 0 & \\
\hline - SCA & 0 & $1(4.3 \%)$ & & 0 & 0 & \\
\hline Potential target large vessel occlusion for EVT & $20 / 75(26.7 \%)$ & $17 / 61(27.9 \%)$ & 1.000 & 9/35 (25.7\%) & $9 / 45(20.0 \%)$ & 0.596 \\
\hline
\end{tabular}

DOAC Direct oral anticoagulant, VKA Vitamin K antagonist, ICA Internal carotid artery, M1 M1-segment of medial cerebral artery, M2 M2-segment of medial cerebral artery, M3 M3-segment of medial cerebral artery, A1 A1-segment of anterior cerebral artery, A2 A2-segment of anterior cerebral artery, V4 V4-segment of vertebral artery, BA Basilar artery, P1 P1-segment of posterior cerebral artery, P2/3 P2 or P3-segment of posterior cerebral artery, SCA Superior cerebellar artery, EVT Endovascular stroke treatment

\section{Discussion}

The comparison of MRI findings in patients with AIS and preceding DOAC versus VKA prescription in our real world university dataset shows the following main findings:

(1) Between DOAC and VKA patients, the frequencies of any LVO (29.3\% versus $37.7 \%, P=0.361)$, and target LVO for endovascular therapy (26.7\% versus $27.9 \%, P=1.0$; aOR 0.835 , 95\% CI 0.368-1.898) were equal with a similar occlusion pattern. (2) Also, the presence of multiple lesions and thrombus characteristics were similar in DOAC and VKA patients. (3) Ischemic lesion diameter in real world patients is equal in patients taking DOAC and VKA and this finding holds true in case of confirmed therapeutic OAC activity. (4) Lesion size in VKA patients was smaller in the setting of confirmed therapeutic VKA. (5) The frequency of radiological hemorrhagic transformation and symptomatic intracranial hemorrhage in OAC patients was low.

AIS in the setting of OAC accounts for about $10 \%$ of all AIS in comprehensive stroke centers with rapidly increasing numbers of preceding DOAC prescriptions due to the increasing number of indications [20, 21]. Besides providing an accurate diagnosis, neuroimaging aids clinicians in patient selection for IVT and EVT as well as early medical management, prognosis assessment and can provide clues to the etiology of the event and hence guide etiological work-up.

Our data show that about 1 in 4 patients with preceding DOAC or VKA has a target LVO for EVT. Macha et al. reported similar frequencies with a decreasing rate of LVO with increasing DOAC plasma levels [7]. Fittingly, Woo et al. reported a LVO rate of 33\% in their cohort of 120 AIS patients with preceding DOAC therapy [8]. The similar occlusion patterns, clot burden and frequency of multiple lesions provide insights into the mechanisms associated with OAC failure and argue for similar stroke etiologies and pathophysiological mechanisms of thrombus formation despite different pharmacological modes of action of preceding VKA and DOAC therapy. In both DOAC and VKA patients, SWI could visualize the thrombus with a higher rate in DOAC (86\%) as compared to VKA patients (57\%). However, due to the small number of patients, this difference in sensitivity to detect the thrombus might be by chance.

Sakamoto et al. found DOAC therapy to be associated with smaller infarct sizes on MRI in a smaller study (40 non-therapeutic VKA, 22 confirmed therapeutic VKA and 29 DOAC, 239 controls) [9]. However, the authors compared non-therapeutic VKA and DOAC treatment with controls without laboratory assessment of DOAC activity or information on compliance. Importantly, the size of infarction for sufficient VKA and DOAC was comparable in their cohort. Oguro et al. also reported smaller infarction sizes in a case series of 7 VKA and 10 DOAC patients with recurrent AIS and also found reduced ischemia volume with DOAC pretreatment [22]. This study was limited by the small sample size and the 
Table 3 Outcome parameter comparing patients with DOAC and VKA pretreatment

\begin{tabular}{|c|c|c|c|c|c|c|}
\hline & $\begin{array}{l}\text { DOAC pretreatment } \\
(\boldsymbol{N}=75)\end{array}$ & $\begin{array}{l}\text { VKA pretreatment } \\
(\boldsymbol{N}=61)\end{array}$ & $P$ & $\begin{array}{l}\text { Confirmed therapeutic } \\
\text { DOAC }(\boldsymbol{N}=35)\end{array}$ & $\begin{array}{l}\text { Confirmed therapeutic } \\
\text { VKA }(\boldsymbol{N}=45)\end{array}$ & $P$ \\
\hline \multicolumn{7}{|l|}{ Clinical outcome } \\
\hline $\mathrm{NIHSS}$ at $24 \mathrm{~h}$ & $2(1-4)$ & $3(0-7)$ & 0.359 & $3(1-5)$ & $4(0-8)$ & 0.565 \\
\hline sICH ECASS-II & $1 / 75(1.3 \%)$ & $1 / 61(1.6 \%)$ & 0.735 & 0 & $1 / 45(2.2 \%)$ & 0.298 \\
\hline Duration of hospital stay, days & $4(2-8)$ & $4(2-8)$ & 0.345 & $4(3-7)$ & $4(2-8)$ & 0.359 \\
\hline $90 \mathrm{~d}$ mRS & $1.5(1-3)$ & $3(1.5-4)$ & 0.001 & $1(1-2)$ & $3(2-6)$ & $<0.001$ \\
\hline $90 \mathrm{~d}$ recurrent stroke & $2 / 75(2.7 \%)$ & $4 / 61(6.6 \%)$ & 0.255 & 0 & $3 / 45(6.7 \%)$ & 0.185 \\
\hline \multicolumn{7}{|l|}{ Imaging } \\
\hline Diameter axis 1 , initial DWI, mm & $18(11-36)$ & $20(7-36)$ & 0.607 & $19(12-33)$ & $13(6-26)$ & 0.111 \\
\hline Diameter axis 2, initial DWI, mm & $10(6-19)$ & $9(5-23)$ & 0.780 & $12(6-19)$ & $7(4-16)$ & 0.127 \\
\hline Diameter hypoperfusion axis 1, mm & $80(38-118), N=40$ & $49(36-67), N=37$ & 0.039 & $83(27-118), N=16$ & $49(36-66), N=13$ & 0.079 \\
\hline Diameter hypoperfusion axis $2, \mathrm{~mm}$ & $34(18-40), N=40$ & $34(14-40), N=37$ & 0.494 & $34(20-46), N=16$ & $33(13-38), N=13$ & 0.318 \\
\hline DWI/Perfusion Mismatch & & & 0.443 & & & 0.714 \\
\hline - None & 39/66 (59.1\%) & 29/53 (54.7\%) & & 17/28 (60.7\%) & $26 / 40(65.0 \%)$ & \\
\hline - Small & 4/66 (6.1\%) & 8/53 (15.1\%) & & $1 / 28(3.6 \%)$ & $3 / 40(7.5 \%)$ & \\
\hline - Medium & $4 / 66(6.1 \%)$ & $3 / 53(5.7 \%)$ & & $2 / 28(7.1 \%)$ & $1 / 40(2.5 \%)$ & \\
\hline - Large & 19/66 (28.8\%) & 13/53 (24.5\%) & & $8 / 28(28.6 \%)$ & $10 / 40(25 \%)$ & \\
\hline Diameter axis 1 , final FLAIR & $18(12-38)$ & $27(8-50)$ & 0.409 & $18(13-23)$ & $20(6-52)$ & 0.945 \\
\hline Diameter axis 2, final FLAIR & $10(6-22)$ & $14(7-25)$ & 0.397 & $10(6-22)$ & $11(5-25)$ & 0.908 \\
\hline Occlusion, any & 22/75 (29.3\%) & 23/61 (37.7\%) & 0.361 & 9/35 (25.7\%) & $14 / 45(31.1 \%)$ & 0.628 \\
\hline Target occlusion for EVT & 20/75 (26.7\%) & $17 / 61$ (27.9\%) & 1.000 & 9/35 (25.7\%) & 9/45 (20.0\%) & 0.596 \\
\hline Thrombus length & $8(6-20), N=19$ & $13(7-24), N=13$ & 0.388 & $15(6-21), N=7$ & $11(4-30), N=6$ & 0.836 \\
\hline Multiple Lesions & $31 / 56(55.4 \%)$ & 24/49 (49.0\%) & 0.560 & 10/21 (47.6\%) & 20/41 (48.8\%) & 1.000 \\
\hline ICH, Heidelberg at follow up & & & 0.024 & & & 0.126 \\
\hline$-1 a$ & $5 / 75(6.7 \%)$ & 3/61 (4.9\%) & & $4 / 35(11.4 \%)$ & $3 / 45(6.7 \%)$ & \\
\hline$-1 b$ & 0 & $7 / 61(11.5 \%)$ & & 0 & $5 / 45(11.1 \%)$ & \\
\hline-2 & 0 & $1 / 61(1.6 \%)$ & & 0 & 0 & \\
\hline$-3 c$ & $1 / 75(1.3 \%)$ & 0 & & $1(2.9 \%)$ & 0 & \\
\hline
\end{tabular}

DOAC Direct oral anticoagulant, VKA Vitamin K antagonist, NIHSS National Institute of Health Stroke Scale, sICH ECASS II symptomatic intracranial hemorrhage according to the European Co-operative Acute Stroke Study-II definition, mRS modified Rankin Scale, DWI Diffusion weighted imaging, FLAIR Fluid attenuated inversion recovery sequence, EVT Endovascular stroke treatment, ICH Intracranial hemorrhage

univariate analysis despite marked baseline differences in CHA2DS2-VASc score, stroke severity of index event and renal dysfunction. Our main finding of equal acute and final infarction diameter in DOAC as compared to VKA patients fits to recently published data on equal clinical stroke severity of patients with preceding DOAC and VKA therapy in real-world patients [16]. Furthermore, the reduced lesion diameter observed in our study in the setting of confirmed VKA therapy is in line with previous findings of smaller ischemic lesions [23] and reduced clinical stroke severity when VKA therapy is within the target range [24]. Macha et al. reported reduced stroke severity in the setting of confirmed DOAC therapy [7]; however our study might have lacked power to prove this difference also in cerebral lesion size.
The low rate of radiological as well as clinically symptomatic intracranial hemorrhage is reassuring; however recanalization strategies were very infrequently used in this cohort.

\section{Strengths and limitations}

The data was collected prospectively, but our study has the inherent limitations of a single-center retrospective analysis. We reliably differentiated between therapeutic and non-therapeutic OAC in patients prescribed VKA and DOAC. For assessment of lesion size, we only measured the diameter and did not use three-dimensional segmentation tools, which does not accurately reflect true ischemic lesion size. Nevertheless, the correlation between maximal diameter and volume seems acceptable [25] and our main focus was the presence of LVO. 


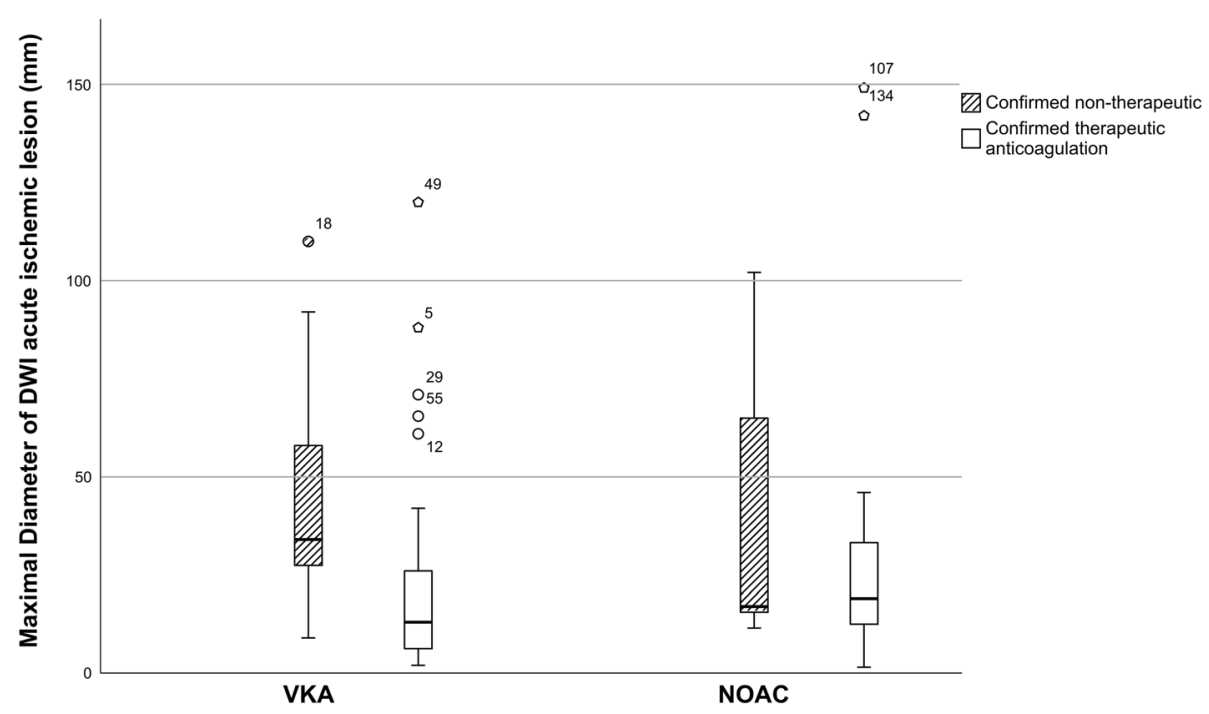

Fig. 1 Lesion Diameter of Acute Ischemic Stroke according to confirmed versus non-therapeutic OAC according to strata of DOAC and VKA. Acute DWI lesion diameter in DOAC patients (median 18, IQR 11-36) as compared to VKA (median 20, IQR 7-36, $P=0.607$ ). Lesion diameter in patients with VKA was significantly lower when OAC was therapeutic (median 13, IQR 6-26 versus median 20, IQR 7-36, $P=0.001$ for MannWhitney-U-Test). NIHSS in patients with DOAC was equal when OAC was therapeutic (median 19, IQR 12-33 versus median 18, IQR 11-36, $P=$ 0.705 for Mann-Whitney-U-Test)

The cutoff of $1 / 3$ used in the mismatch analysis was arbitrary and might not be easily comparable to more commonly used cutoffs such as $>20 \%$ of the ischemic lesion. Although baseline characteristics of the DOAC and VKA group were almost identical, we might have missed confounding baseline variables influencing the physicians' indication of DOAC/VKA and affecting imaging outcomes. Due to the limited number of patients with DOAC other than Rivaroxaban, our findings should not be extrapolated to those substances. Therefore, the findings have to be replicated in further cohorts.

\section{Conclusion}

In conclusion, we found that about 1 in 4 patients with preceding OAC has a target LVO for EVT. The ischemic lesion size was equal in patients taking DOAC and VKA, also in the setting of confirmed therapeutic OAC activity. Since imaging features are similar, the mechanisms associated with AIS despite preceding DOAC or VKA treatment seem alike.

\section{Abbreviations}

ADC: Apparent Diffusion Coefficient; AF: Atrial Fibrillation; AIS: Acute Ischemic stroke; aOR: adjusted Odds Ratio; CMB: Cerebral Microbleeds; DOAC: Direct Oral Anticoagulants; DWI: Diffusion-Weighted Imaging; DSC: Dynamic Susceptibility Contrast; EVT: Endovascular Therapy; FLAIR: Fluid-Attenuated Inversion Recovery; INR: International Normalized Ratio; IVT: Intravenous Thrombolysis; LVO: Large Vessel Occlusion; MRI: Magnetic Resonance Imaging; NIHSS: National Institutes of Health Stroke Scale; OAC: Oral Anticoagulation; SWI: Susceptibility-Weighted Imaging; TOF: Time of Flight; VKA: Vitamin K Antagonists

\section{Acknowledgements}

None.

\section{Authors' contributions}

Senior authors SJ and AH contributed equally to this paper. TRM was involved in the study design, wrote the statistical analysis plan, organized and analyzed the data, and drafted and revised the paper. JK, EA, SF were responsible for clinical data acquisition, designed data collection tools, drafted and revised the paper. JG, DJS, ME-K, MA, UF monitored data collection of the registry, was involved in study design and made critical revision of the manuscript. MA, UF, MG, SJ and AH contributed in the design of the registry, and made critical revision of the manuscript. JK provided statistical advice. The author(s) read and approved the final manuscript.

\section{Funding}

None.

\section{Availability of data and materials}

The datasets used and/or analysed during the current study are available from the corresponding author on reasonable request.

\section{Ethics approval and consent to participate}

The competent ethics committee approved this single-center observational study (Kantonale Ethikkommission Bern, reference number KEK 231/14). Verbal consent was obtained according to Swiss legislation and approved by the ethics committee. Patients who withdrew their consent were excluded from the data export.

\section{Consent for publication}

Not applicable.

\section{Competing interests}

Dr. Gralla received Swiss National Science Foundation (SNSF) grants for magnetic resonance imaging in stroke. Professor Arnold received speeking fees and honoraria for scientific advisory boards from Covidien and Medtronic. Dr. Fischer is a consultant for Medtronic and Stryker. Mr. Kaesmacher has received travel grants from Pfizer and Stryker. He also is an Editorial Board Member of BMC Neurology. All other authors have nothing to disclose.

\section{Author details}

'Department of Neurology, Inselspital, Bern University Hospital, University of Bern, Freiburgstrasse 8, CH-3010 Bern, Switzerland. ${ }^{2}$ Institute of Diagnostic and Interventional Neuroradiology, Institute of Diagnostic, Interventional and 
Pediatric Radiology and Department of Neurology, University Hospital Bern Inselspital, University of Bern, Bern, Switzerland. ${ }^{3}$ University Institute of Diagnostic and Interventional Neuroradiology, Inselspital, Bern University Hospital, University of Bern, Bern, Switzerland.

\section{Received: 4 December 2019 Accepted: 6 March 2020}

Published online: 11 March 2020

\section{References}

1. Kirchhof P, Benussi S, Kotecha D, Ahlsson A, Atar D, Casadei B, et al. 2016 ESC guidelines for the management of atrial fibrillation developed in collaboration with EACTS. Eur Heart J. 2016;37(38):2893-962..

2. Ruff CT, Giugliano RP, Braunwald E, Hoffman EB, Deenadayalu N, Ezekowitz $M D$, et al. Comparison of the efficacy and safety of new oral anticoagulants with warfarin in patients with atrial fibrillation: a meta-analysis of randomised trials. Lancet. 2014;383(9921):955-62.

3. Almutairi AR, Zhou L, Gellad WF, Lee JK, Slack MK, Martin JR, et al. Effectiveness and safety of non-vitamin $\mathrm{K}$ antagonist oral anticoagulants for atrial fibrillation and venous thromboembolism: a systematic review and meta-analyses. Clin Ther. 2017;39(7):1456-1478.e36.

4. El-Koussy M, Schroth G, Brekenfeld C, Arnold M. Imaging of acute ischemic stroke. Eur Neurol. 2014:72(5-6):309-16.

5. Menon BK, Campbell BCV, Levi C, Goyal M. Role of imaging in current acute ischemic stroke workflow for endovascular therapy. Stroke. 2015;46(6):1453-61.

6. Tong E, Hou Q, Fiebach JB, Wintermark $M$. The role of imaging in acute ischemic stroke. Neurosurg Focus. 2014;36(1):1-17. https://doi.org/10.3171/ 2013.10.FOCUS13396.

7. Macha K, Marsch A, Siedler G, Breuer L, Strasser EF, Engelhorn T, et al. Cerebral ischemia in patients on direct Oral anticoagulants. Stroke. 2019;50(4):873-9. https://doi.org/10.1161/STROKEAHA.118.023877.

8. Woo HG, Chung I, Gwak DS, Kim BK, Kim BJ, Bae HJ, et al. Recurrent ischemic stroke in atrial fibrillation with non-vitamin $\mathrm{K}$ antagonist oral anticoagulation. J Clin Neurosci. 2019;64:127-33. Available from. https://doi. org/10.1016/j.jocn.2019.03.037.

9. Sakamoto Y, Okubo S, Sekine T, Nito C, Suda S, Matsumoto N, et al. Prior direct Oral anticoagulant therapy is related to small infarct volume and no major artery occlusion in patients with stroke and non-Valvular atrial fibrillation. J Am Heart Assoc. 2018;7(17):1-7. Available from. https://doi.org/ 10.1161/JAHA.118.009507.

10. Kanai Y, Oguro H, Tahara N, Matsuda H, Takayoshi H, Mitaki S, et al. Analysis of recurrent stroke volume and prognosis between warfarin and four nonvitamin K antagonist Oral anticoagulants' Administration for Secondary Prevention of stroke. J Stroke Cerebrovasc Dis. 2018;27(2):338-45.

11. Lim MS, Chapman K, Swanepoel P, Enjeti AK. Sensitivity of routine coagulation assays to direct oral anticoagulants: patient samples versus commercial drug-specific calibrators. Pathology. 2016;48(7):712-9.

12. Ten Cate $\mathrm{H}$, Henskens YM, Lancé MD. Practical guidance on the use of laboratory testing in the management of bleeding in patients receiving direct oral anticoagulants. Vasc Health Risk Manag. 2017;13:457-67.

13. Toyoda K, Yamagami H, Koga M. Consensus guides on stroke thrombolysis for Anticoagulated patients from Japan: application to other populations. J stroke. 2018;20(3):321-31.

14. Douxfils J, Ageno W, Samama CM, Lessire S, ten Cate H, Verhamme P, et al. Laboratory testing in patients treated with direct oral anticoagulants: a practical guide for clinicians. J Thromb Haemost. 2018;16(2):209-19.

15. Touzé E, Gruel Y, Gouin-Thibault I, De Maistre E, Susen S, Sie P, et al. Intravenous thrombolysis for acute ischemic stroke in patients on direct oral anticoagulants. Eur J Neurol. 2018;25(5):747-e52.

16. Auer E, Frey S, Kaesmacher J, Hakim A, Seiffge DJ, Goeldlin M, et al. Stroke severity in patients with preceding direct oral anticoagulant therapy as compared to vitamin K antagonists. J Neurol. 2019;266(9):2263-72.

17. Greenberg SM, Vernooij MW, Cordonnier C, Viswanathan A, Al-Shahi Salman R, Warach S, et al. Cerebral microbleeds: a guide to detection and interpretation. Lancet Neurol. 2009;8(2):165-74.

18. Von Kummer R, Broderick JP, Campbell BCV, Demchuk A, Goyal M, Hill MD, et al. The Heidelberg bleeding classification: classification of bleeding events after ischemic stroke and reperfusion therapy. Stroke. 2015;46(10):2981-6.

19. Wahlund LO, Barkhof F, Fazekas F, Bronge L, Augustin M, Sjögren M, et al. A new rating scale for age-related white matter changes applicable to MRI and CT. Stroke. 2001;32(6):1318-22.
20. Meinel TR, Kaesmacher J, Chaloulos-lakovidis P, Panos L, Mordasini P, Gralla $J$, Fischer $U$. Clinical effect of successful reperfusion in patients presenting with posterior circulation large vessel occlusion: Data from a multicenter registry. Zurich: 22 Jahrestagung der Schweizerischen Hirnschlaggesellschaft 2019 SHG-SCS; 2019

21. Seiffge DJ, Kägi G, Michel P, Fischer U, Béjot $Y$, Wegener $S$, et al. Rivaroxaban plasma levels in acute ischemic stroke and intracerebral hemorrhage. Ann Neurol. 2018;83(3):451-9.

22. Oguro H, Mizuhara R, Abe S, Takayoshi H, Mitaki S, Onoda K, et al. Analysis of recurrent stroke volume between VKA (Vitamin K Antagonist) and three NOACs (non-vitamin K antagonist oral anticoagulants) under oral anticoagulant therapy. J Stroke Cerebrovasc Dis. 2018;27(2):338-45. https:// doi.org/10.1016/j.jstrokecerebrovasdis.2017.09.007. Epub 2017 Oct 13.

23. Ay $H$, Arsava EM, Gungor L, Greer D, Singhal AB, Furie $K L$, et al. Admission international normalized ratio and acute infarct volume in ischemic stroke. Ann Neurol. 2008;64(5):499-506.

24. Hylek EM, Go AS, Chang Y, Jensvold NG, Henault LE, Selby JV, et al. Effect of intensity of Oral anticoagulation on stroke severity and mortality in atria fibrillation. N Engl J Med. 2003;349(11):1019-26.

25. Gillard J, Waldman A, Barker P. (Eds). Clinical MR Neuroimaging: Physiological and Functional Techniques. Cambridge: Cambridge University Press; 2019. https://doi.org/10.1017/CBO9781139193481.

\section{Publisher's Note}

Springer Nature remains neutral with regard to jurisdictional claims in published maps and institutional affiliations.
Ready to submit your research? Choose BMC and benefit from:

- fast, convenient online submission

- thorough peer review by experienced researchers in your field

- rapid publication on acceptance

- support for research data, including large and complex data types

- gold Open Access which fosters wider collaboration and increased citations

- maximum visibility for your research: over $100 \mathrm{M}$ website views per year

At BMC, research is always in progress.

Learn more biomedcentral.com/submissions 